\title{
Chronobiological theories of mood disorder
}

Nevin F.W. Zaki ${ }^{1, C A}$, David Warren Spence' ${ }^{2}$,Ahmed S. BaHammam³, Seithikurippu R. Pandi-Perumal ${ }^{4}$, Daniel P. Cardinalii ${ }^{5}$, Gregory M. Brown ${ }^{6}$

${ }^{1}$ Department of Psychiatry, Faculty of Medicine, Mansoura University, Mansoura, Egypt.

2652 Dufferin Street, Toronto, ON M6K 2B4, Canada.

${ }^{3}$ University Sleep Disorders Center, College of Medicine, King Saud University, Riyadh, Saudi Arabia.

${ }^{4}$ Somnogen Canada Inc, Toronto, ON, Canada.

${ }^{5}$ BIOMED-UCA-CONICET and Department of Teaching and Research, Faculty of Medical Sciences, Pontificia Universidad Católica Argentina, Buenos Aires, Argentina

${ }^{6}$ Centre for Addiction and Mental Health, Department of Psychiatry, University of Toronto, ON M5T 1R8, Canada

\section{Corresponding author}

Nevin F.W. Zaki

Assistant Professor of Psychiatry

Department of Psychiatry

Faculty of Medicine

Mansoura University Mansoura,

Egypt

Tel: +201283339789

mernakero@mans.edu.egor

nevinfzaki@yahoo.com

Running head: Sleep and depression 


\section{Abstract}

Background: Major depressive disorder (MDD) remains the most prevalent mental disorder and a leading cause of disability, affecting approximately 100 million adults worldwide. The disorder is characterized by a constellation of symptoms affecting mood, anxiety, neurochemical balance, sleep patterns, and circadian and/or seasonal rhythm entrainment. However, the mechanisms underlying the association between chronobiological parameters and depression remain unknown.

Methods: A PubMed search was conducted to review articles from 1979 to the present, using the following search terms: "chronobiology," "mood," "sleep," and "circadian rhythms." We aimed to synthesize the literature investigating chronobiological theories of mood disorders.

Results: Current treatments primarily include tricyclic antidepressants and selective serotonin reuptake inhibitors, which are known to increase extracellular concentrations of monoamine neurotransmitters. However, these antidepressants do not treat the sleep disturbances or circadian and/or seasonal rhythm dysfunctions associated with depressive disorders. Several theories associating sleep and circadian rhythm disturbances with depression have been proposed. Current evidence supports the existence of associations between these, but the direction of causality remains elusive.

Conclusions: Given the existence of chronobiological disturbances in depression and evidence regarding their treatment in improving depression, a chronobiological approach, including timely use of light and melatonin agonists, could complement the treatment of MDD. 


\section{Key words}

Chronobiology; circadian rhythm; major depressive disorder; mood disorder; light environment; melatonin 


\section{Introduction}

Sleep and circadian rhythm disturbances frequently occur in major depressive disorder (MDD) and tend to persist across the different phases of the disorder (prodrome, acute episodes, and remission periods). These disturbances can precipitate depression or exacerbate an existing depressive disorder and may also increase resistance to treatment. The suprachiasmatic nucleus (SCN) of the anterior hypothalamus is the major circadian pacemaker of the body and is entrained to a 24h circadian rhythm by environmental zeitgebers (time cues).

There is robust evidence of disrupted circadian rhythms in depression, which has led to several hypotheses to explain this association. However, fundamental questions have been raised regarding the direction of causality between disturbed sleep/wake cycle and mood disorders. Such questions have both theoretical and practical relevance for understanding how depressive disorders evolve, and given the frequent co-occurrence of circadian disruption of sleep with depressive symptoms, answers to these questions could ultimately improve the prognosis and treatment of patients with depression.

Given the importance of sleep and chronobiological issues in clinically managing mood disorders, understanding the various interactions between sleep and mood is essential for practicing clinicians. In this review, we summarize this complex relationship using chronobiological theories of mood disorders. To provide a comprehensive overview of the literature, a PubMed search was conducted to review articles from 1979 to date, using the following search terms: "chronobiology," "mood," "sleep," and "circadian rhythms." This study aimed to review the literature with regards to studies investigating chronobiological theories of mood disorders. 


\section{The circadian pacemaker}

The master clock controlling chronophysiological rhythms of the brain and body organs is located in the SCN. This neural structure was first recognized as a discrete group of neurons in the 1970s (1-3). The SCN contains local projection neurons that communicate with one another and with other hypothalamic structures (4). The axons of many SCN neurons terminate within the nucleus itself, thus forming local circuit connections and/or collaterals from longer-range projections. The SCN core projects densely to the $\mathrm{SCN}$ shell, which has sparse projections back to the core. Neuronal cell bodies in the SCN are small ( $10 \mu \mathrm{M})$, have simple dendritic arbors, and are closely apposed.

Neurons in the SCN core and peripheral regions differ on the basis of their neurochemical content. The neuropeptide vasoactive intestinal peptide (VIP) is expressed in approximately $10 \%$ of all SCN neurons, while arginine vasopressin (AVP) is expressed in approximately $20 \%$ of SCN neurons $(5,6)$. VIP-positive neurons are mainly located in the ventral and central part of the SCN (i.e., the core). In humans, the volume of the VIP core subdivision is $0.03 \mathrm{~mm}^{3}$ and contains about 1,700 VIPimmunoreactive neurons, with a mean density of about 63,000 neurons $/ \mathrm{mm}^{3}$ (7). In addition to VIP, neurons in the SCN core also contain substance $\mathrm{P}$, gastrin-releasing peptide, calretinin, and calbindin. The largest proportion of AVP-positive neurons is in the dorsomedial part of the SCN (i.e., the shell). In humans, the volume of the AVP subdivision is $0.2 \mathrm{~mm}^{3}$ and contains about 6900 AVP-immunoreactive neurons, with a mean density of 29,000 neurons $/ \mathrm{mm}^{3}$. In this region, neurons containing cholecystokinin (CCK) and prokineticin 2 are found in addition to AVP neurons. 
In most SCN neurons, neuropeptides are co-localized with gamma-aminobutyric acid (GABA) and almost all synapses among SCN neurons are GABAergic. It has been reported from electrophysiological data that glutamate is also present in efferent pathways of the SCN.

Increased electrical activity in the SCN during the day has been observed in both nocturnal mammals, such as the hamster or the rat, and diurnal species, such as the primates. However, in primates, at the beginning of the light phase there is secretion of corticosteroids, the onset of activity and phase of sympathetic predominance, and a rise in body temperature, whereas these occur at the beginning of the dark phase in rats. This shows that the signal produced by the SCN to different effectors (mentioned above) is interpreted in different ways.

Synchronization to the natural environmental light/dark (LD) cycle occurs through retinal light perception. Humans' endogenous rhythmic activity has a period slightly longer than $24 \mathrm{~h}$; hence, it needs daily entrainment to $24 \mathrm{~h}$ with zeitgebers (regularly repetitive environmental signals). Light is the most pervasive and prominent zeitgeber for the SCN (8). Rod and cone cells in the retina play a relatively minor role in "non-visual" circadian photic input. However, the primary photoreceptor pigment involved in circadian rhythmicity is melanopsin, present in a minute group of uniquely photosensitive retinal ganglion cells (9). These cells form the specialized retinohypothalamic tract (RHT), which has efferent connections to the SCN, as well as with nuclei mediating pupillary responses. The $\mathrm{SCN}$, in turn, receives other non-photic inputs (e.g., from serotonin (5-HT)-containing neurons in the raphe nuclei) (10). 
The SCN drives and controls nocturnal synthesis and secretion of the pineal hormone melatonin, which in turn interacts with melatonin receptors on SCN neurons (11). The master SCN clock regulates secondary oscillators present in most of the body's organs. Consequently, most physiological functions display rhythmic changes. Furthermore, this action extends to the cyclical, ebb-and-flow activity of most mental and emotional functions, e.g., stupor, depression, elation, and excitement (12).

\section{CLOCK genes and depression}

There has been increasing evidence that CLOCK genes regulate mood. There are more than ten clock genes, which interact to produce circadian rhythmic activity within the $\mathrm{SCN}^{1,2}$. Evidence has now been presented that the circadian genes BMAL1, Period3, and Timeless are associated with MDD and bipolar disorder (13). Genotypic polymorphisms of CLOCK genes may affect the core symptomatology of mood disorders. A single nucleotide polymorphism (SNP) in the 3-flanking region of CLOCK correlates with sleep profiles, rest/activity rhythms, and relapse of depressive episodes (14). SNPs are the most common type of genetic variation among humans. For example, an SNP may appear as the replacement of the nucleotide cytidine monophosphate (CMP) with the nucleotide thymidine monophosphate (TMP) in a certain sequence of DNA. SNPs usually occur throughout a person's DNA, on average once in every 300 nucleotides, meaning there are roughly 10 million SNPs in the human genome. Most commonly, these variations are found in intergenic spaces. At these locations they can act as biological markers, helping to locate genes associated with a disease. In contrast, when SNPs occur 
within a gene or in a regulatory region near a gene, they may play a more direct role in disease by affecting the gene's function (15).

It has been reported that a rare genetic polymorphism in Period3 affects response to pharmaceutical antidepressant treatment, particularly selective 5-HT reuptake inhibitors (SSRIs). A variable-number tandem-repeat polymorphism is contained in the coding region of the Per3 gene, which encodes 18 amino acids, and is repeated either four (Per34) or five (Per35) times. These repeated units contain phosphorylation sites, which in turn affect Per3 function (15). Studies investigating circadian phenotypes have shown that in healthy individuals Per35 was associated with a "morningness" chronotype and increased slow wave activity (SWA; often referred to as deep sleep, consisting of stage three of non-rapid eye movement sleep, N3)(16). In contrast, Per34 was associated with an "eveningness" chronotype and delayed sleep phase disorder (DSPS). These discoveries, along with several others, have led researchers to consider circadian genes as beneficial endophenotypes of mood disorders (17-19).

It is now known that brain 5-HT synthesis, release, and catabolism follow a circadian rhythm, and are closely connected to the SCN. Indeed, an endogenous circadian rhythm in neuronal 5-HT release in the SCN occurs in the absence of photic cues. In a study by de Pontes et al., it was observed that the level of 5-HT started to increase in the raphe nuclei immediately after the onset of the dark phase. The raphe itself displays its own circadian features, such as tryptophan hydroxylase (TpH) activity within the nucleus. This enzyme is a limiting step in the synthesis of 5-HT. Peak expression of $\mathrm{TpH}$ is observed during the dark phase (20)'(21), providing neuroanatomical evidence that the $5-\mathrm{HT}$ system interacts with the master body clock 
(22). Further supporting evidence comes from findings that serotonergic neurotransmission influences the enzymatic phosphorylation of CLOCK proteins that constitute the molecular oscillator, and leads to phase shifts and entrainment of SCN activity (23). Furthermore, it was reported that the SSRI antidepressant fluoxetine altered circadian timekeeping by causing phase advancing of neuronal firing of the SCN (23).

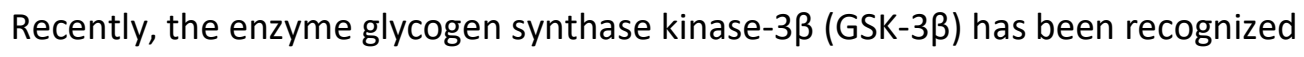
as a key controller of the circadian clock. GSK-3 $\beta$ is a serine/threonine kinase that controls glycogen metabolism. The phosphorylation of PER2 by GSK-3 $\beta$ leads to a buildup of PER2 in the SCN. There, it enhances NPAS2/BMAL1-mediated transcription with an increasing production of enzymes in the inner membranes of mitochondria. It has been suggested that increased levels of PER2 might lead to dopamine deficiencies and a more depressed mood state (24). The action of GSK-3 $\beta$ is inhibited by the mood stabilizer lithium, which lengthens the period of the biological clock, an effect thought to explain its effectiveness in bipolar disorder in those with shortened rhythms ${ }^{3}$. Conversely, GSK-3 $\beta$ decreases the phosphorylation of PER2, causing PER2 to become scarce in the SCN and leading to decreased MAOA production. This is associated with increased dopamine production and could lead to corresponding improvements in mood (25). Table 1 provides a summary of 15 circadian genes tested for associations with bipolar disorder (adapted from Shi et al. (26)). 
Table 1 Circadian genes that have been investigated in association with bipolar disorder

\begin{tabular}{|c|c|c|}
\hline GENE NAME & Full Gene Name & FUNCTION \\
\hline CRY2 & cryptochrome 2 & inhibition of CLOCK-BMAL \\
\hline PER1 & period 1 & inhibition of CLOCK-BMAL \\
\hline PER2 & period 2 & inhibition of CLOCK-BMAL \\
\hline PER3 & period 3 & association WITH CRY \\
\hline \multirow{2}{*}{ TIMELESS } & \multirow{2}{*}{ timeless homolog drosophilia } & interact WITH PER1 \\
\hline & & inhibit CLOCK/BMAL INUCED TRANSACTIVATION \\
\hline ARNTL1(BMAL1) & $\begin{array}{l}\text { aryl hydrocarbon receptor } \\
\text { nuclear translocate like } 1\end{array}$ & activate CLOCK \&CLOCK controlled genes \\
\hline ARNTL2(BMAL2) & $\begin{array}{l}\text { aryl hydrocarbon receptor } \\
\text { nuclear translocate like } 1\end{array}$ & activate CLOCK \&CLOCK controlled genes \\
\hline BHLHB2 & $\begin{array}{c}\text { basic helix loop helix domain } \\
\text { containing class B2 }\end{array}$ & inhibit CLOCK/BMAL INUCED TRANSACTIVATION \\
\hline BHLHB3 & $\begin{array}{l}\text { basic helix loop helix domain } \\
\text { containing class B3 }\end{array}$ & inhibit CLOCK/BMAL INUCED TRANSACTIVATION \\
\hline CLOCK & $\begin{array}{l}\text { circadian locomotor output } \\
\text { cycles protein kaput }\end{array}$ & activate CLOCK \&CLOCK CONTROLLED GENES \\
\hline CRY1 & & inhibition of CLOCK-BMAL \\
\hline CSNK1D & casein kinase 1 delta & phosphorylate PER,GRY,BMAL \\
\hline CSNK1E & casein kinase 1 epilson & phosphorylate PER,GRY,BMAL \\
\hline DBP & $\begin{array}{l}\text { site of albumin promotor } \\
\text { binding protein }\end{array}$ & output activation OF PER1 \\
\hline NR1D1(REVERBA) & $\begin{array}{c}\text { nuclear receptor subfamily } 1 \\
\text { group D member } 1\end{array}$ & INHIBITION OF BMAL \\
\hline
\end{tabular}

This table is adapted from Shi et al. (2008). A similar table could be produced for MDD.

\section{Circadian desynchronization}

The occurrence of circadian rhythm disruption in MDD was first described over 20

years ago (27). This well-documented finding is further reinforced by the inclusion of insomnia and hypersomnia as one of the nine defining diagnostic criteria for major depressive episodes in the Diagnostic and Statistical Manual of Mental Disorders 5 (DSM 5) (28). Additionally, there is robust evidence of a bidirectional link between sleep disturbances and depression, with insomnia now recognized as a predisposing 
factor for developing depression (29). There is evidence that depression itself can alter sleep architecture in various ways. Nutt et al.(30) reported that depression can lead to lower sleep efficiency, increased sleep onset latency, and frequent and longer periods of wakefulness. Furthermore, the presence of depressive symptoms shortens slow wave sleep (SWS) and leads to several changes to rapid eye movement (REM) sleep, including shorter REM latencies, an increased length of the first REM period, and an increased quantity of eye movements (REM density) (31). These sleep abnormalities may lead to relapse and a decreased response to therapeutic interventions.

The co-occurrence of depression and sleep disturbance may be a physiological reaction to a more definitive disruption in circadian rhythms, i.e., circadian disruption could be both an antecedent and causal primary condition for the development of depressive symptoms $(32,33)$. Alternatively, sleep disruption and depressive illness may essentially be independent phenomena, but nevertheless have reciprocal causal effects, and possibly signify an interruption in feedback mechanisms that usually distinguish their interaction. It has been suggested that this latter hypothesis is consistent with the view that both of these pathological processes take place concurrently (32).

Van den Hoofdakker (34) reviewed the circadian basis of mood disorders and proposed several theories based on observational studies of individuals entraining to $24 \mathrm{~h}$ environmental time cues, particularly the LD cycle. Particular chronobiological properties of the circadian system, such as the acrophase, period, amplitude, and its interactions with the ultradian NREM sleep-REM sleep cycle, as well as phase relations with sleep-wake rhythms, are best studied within certain protocols 
including free running forced desynchronization or constant routines. These various parameters have been studied in rats, but certain elements have now also been applied to human models of depression (35).

The two-process model of sleep, first proposed by Borbély in 1982, explains how homeostatic and circadian factors regulate the quantity and timing of sleep(36) (Fig. 1). According to this view, the requirement for sleep increases during wakefulness because of homeostatic process $\mathrm{S}$ in the brain, while circadian process $\mathrm{C}$ reflects circadian modification of vigilance. Borbély's theory states that the likelihood of wakefulness and sleep are traded off against one another in a circadian mode. Homeostatic process $\mathrm{S}$ is defined as a homeostatic sleep-promoting process, which continuously escalates during the wakeful period. Process $S$ is related to decreased intellectual performance and vigilance, and an increase in sleepiness/fatigue while awake. During sleep, particularly non-REM or SWS, process S continuously decreases (i.e., sleep pressure disintegrates). In contrast, the circadian scheduling process $C$ (also known as the circadian pacemaker) is best seen as a nearly $24 \mathrm{~h}$ endogenous oscillatory variation for sleep propensity.

Circadian-based vigilance propensity is at its lowest level during the early evening hours, during which homeostatic sleep pressure is elevated, and reaches its highest level during the early morning, during which homeostatic sleep pressure is low. Process $\mathrm{C}$ is a clock-like process that is independent of whether the subject is asleep or awake, and is synchronized with external or environmental time under normal conditions. In humans, the phase of the endogenous circadian pacemaker (process C) can be reliably determined from the rhythmic changes in endogenous core body temperature or melatonin secretion from the pineal gland. In tandem with the 
circadian phase, endogenous melatonin secretion reaches its nadir at the time of maximal circadian vigilance propensity, and peaks at the time of minimal circadian vigilance propensity. Core body temperature essentially shows the reverse pattern. These rhythmic changes in process $\mathrm{C}$ are organized by the SCN (2).

Two types of data support the main premise of chronobiological theories of depression. The first comprises conventional phenomena, such as diurnal fluctuations in depressive states, early morning awakening, and, in some individuals, seasonal discrepancy of onset. Diurnal variation implies circadian variations in the clinical state, with severity of symptoms appearing to be associated with either the time of the day or to the sleep-wake cycle. Early morning awakening might be a manifestation of pathologically advanced timing in the rhythm of sleep propensity. It has been suggested that recurrent annual regularity, which occurs in some patients with bipolar disorder, provides evidence of its fundamentally photoperiodic nature (37).

The second type of evidence supporting the hypothesis that mood states are heavily influenced by circadian functioning comes from studies demonstrating the antidepressant effects of chronobiologic manipulations, i.e., light/melatonin exposure and the sleep/wake cycle (Fig. 1). There has been debate about the circadian control of the sleep/wake cycle. Most studies support the conclusion that the rest/activity and sleep/wake cycles are affected by a range of factors, and are thus less consistent in their capacity for entrainment than other rhythms, such as core body temperature, cortisol and melatonin release, REM sleep propensity, alertness, and cognitive functions. Within the framework of the two-process model 
(36), Daan and Beersma have provided an objectively valid consideration of the influence of NREM and sleep timing on mood (Fig. 1) (38).

\section{Dysregulation of the endogenous clock system}

Several clinical observations support the suggestion that the various expressions of mood disorders may result from circadian dysregulation (39). For instance, it has been noted that some patients with MDD undergo predictable recurrent cycles of altered mood, and that many patients with MDD have marked diurnal mood variation (depressed mood is usually more severe in the morning). Other patients with depression display abnormalities in phase timing, with a phase-advanced elevation of nocturnal body temperature occurring over the $24 \mathrm{~h}$ diurnal cycle (rhythms in healthy controls show higher values in the evening, followed by progressive temperature reduction, with the nadir occurring in the middle of the night).

Another symptomatic feature now recognized in many patients with depression relates to cortisol, with affected individuals showing increased cortisol secretion and a phase advance in its rhythm, especially among those with a melancholic subtype. In contrast, in healthy individuals, maximal secretion of cortisol occurs in the morning, followed by a progressive reduction throughout the day until a nadir is reached in the evening (40). Patients with major depression also often show lower blood concentrations of melatonin with pronounced circadian phase advances in melatonin secretion. However, melatonin secretion is inhibited by light exposure. Therefore, its concentration may be affected by disturbed sleep and exposure to light at inappropriate times. Furthermore, melatonin secretion in patients with 
bipolar disorder and their offspring has been shown to exhibit a modified sensitivity to the suppressant effect of light than do healthy individuals (41).

Most individuals with MDD report experiencing subjective changes in their sleep/wake cycles, which have been documented to occur in parallel with abnormalities in sleep architecture. Even among patients in whom most symptoms of depression are in remission, residual sleep abnormalities have been shown to recur.

Patients with seasonal affective disorder experience episodes of major depression that often occur in the fall or winter. The observed associations between mood disorders and sleep abnormalities have led to the development of therapeutic strategies targeting this relationship. Light therapy and sleep deprivation are now well-established interventions with confirmed antidepressant effects (42) (Fig. 1). The effects of mood stabilizers on the rhythmicity of the endogenous clock provide further evidence of the link between circadian rhythms and mood. For example, the dual-acting drug agomelatine is thought to target melatonergic receptors to reestablish disrupted circadian rhythms, but also simultaneously elevates depressed mood, probably via $5-\mathrm{HT}_{2}$ receptor antagonism. Agomelatine was initially considered the first of what might become a new generation of antidepressants, whose mechanism of action included a "circadian stabilizer" (3). However, it is now known that the proposed chronobiologic basis underlying the effect of agomelatine was incorrect. However, the phase resetting action of melatonin and melatonergic drugs generally acts via a well-defined phase response curve that requires an extremely short-lived pulse and not a long-acting one such as that produced by 
agomelatine ${ }^{4,5}$. Agomelatine has also been shown to be hepatotoxic in some patients and therefore should be used with caution ${ }^{5,6}$.

\section{Chronobiological models of mood disorders}

Four major models have been proposed to describe the chronobiological basis of mood disorders. Each of these is outlined below.

\section{Internal coincidence model}

The first model is the internal coincidence model developed by Wehr and WirzJustice (43). This model asserts that the phase angle difference between the SCN and the sleep/wake cycle is depressogenic. In other words, the model asserts that sleep can prompt depression when it is not synchronized (or coincident) with a critically relevant circadian phase. According to the model, patients with depression sleep at a times inappropriate for their biological clock (similar to shift workers who sleep during the day or jet-lagged travelers who are suddenly forced to sleep in a different environmental time zone). It has been assumed that depression arises when circadian oscillators are phase advanced compared with environmental zeitgebers.

Furthermore, according to the model, depression occurs when particular circadian functions are phase shifted with respect to each other, for example in shiftbased occupations. A phase shift in circadian rhythms means that bedtime and waking time move earlier in the day (phase advance) or later in the day (phase delay) (44). In this pathological state, a prolonged period of desynchronization of circadian cycles of various bodily processes (e.g., temperature cycles, metabolic rate, melatonin secretion, etc.) is associated with stress and phase instability.

\section{Shift in circadian cycle peaks}


The second model is the shift or drifting of the peak of one or more individual circadian cycles away from their normal relationship with other cycles. This hypothesis was supported by observations that an advancing sleep episode in patients with depression (reducing the mismatch in circadian and sleep phases) was associated with improved mood. Similarly, antidepressant medications, such as monoamine oxidase inhibitors and mood stabilizers, were reported to extend the endogenous circadian period in patients with mood disorders (45).

Later, Reinberg and Ashkenazi reinterpreted the model to consider the influence of other factors, such as changes in rhythm (prominent period; $\tau$ ) and period instability (46). According to this revised view, the endogenous circadian system is advanced in relation to the sleep timetable, such that deep sleep (SWS) occurs during circadian phases near the end of the night. This disruption of the normal cycling of sleep stages exerts a depressogenic influence in susceptible individuals. The rescheduling of sleep to 5 or 6 hours earlier than an individual's normal retiring time has been successful in ameliorating depressive symptoms. Nevertheless, REM sleep onset latency and duration still remain abnormal even after the sleep period has been advanced; thus, sleep rescheduling does not consistently produce the expected antidepressant effect (47).

Studies comparing depressed and non-depressed individuals have supported the inference that advanced circadian phase phenomena, such as early morning awakenings, accelerated onset of REM sleep in relation to sleep onset, and shift of melatonin secretion, are attributable to a phase shift in the central circadian oscillator. Phase shift hypotheses have driven the development of therapeutic 
methods using light therapy and intake of melatonin to resynchronize circadian rhythms and the sleep/wake cycle of patients with depression. Such therapies have generated positive and encouraging results, particularly in patients with seasonal affective disorder (“winter depression") (48).

The results of several decades of research of therapeutic interventions with patients with depression have shown that efforts to reduce misalignment in circadian and sleep phases are in fact associated with improvements in mood in affected patients. Thus, these provide objective confirmation of the internal coincidence model. Further confirmatory evidence has come from clinical and experimental studies of antidepressant medications, such as monoamine oxidase inhibitors and mood stabilizers, which have been found to prolong parallel changes in the circadian periods of patients with mood disorders. However, a comparison of the circadian periods in depressed and healthy individuals failed to reliably support the phase-advance hypothesis (45). Nevertheless, most of the evidence provided to date does support the inference of a link between disrupted circadian processes and depressed mood; however, the manner in which this occurs is yet to be investigated. Moreover, the reasons for the failure of the internal coincidence model to account for some inconsistencies in its predictions remain unclear. However, the evidence does generally support the proposal that desynchronization of circadian rhythms may lead to a depressed state (3).

\section{S-deficiency model}

Another model to be considered is the S-deficiency model, first proposed by Borbély (49).The model is actually an extension of the two-process model of sleep regulation, although no presumption of circadian dysregulation is made, i.e., it is assumed that 
process $C$ is unaffected and that the core disruption is the absence of process $S$. This model proposes that the level of S during the waking period of patients with depression does not rise to the level which is reached in those without depression (36). Thus, the buildup of Process $S$ becomes deficient.

This hypothesis relies on two assumptions. First, the S-deficiency is assumed to explain sleep disturbances in depression. Second, a causal link between the level of S and depressive symptomatology is postulated to account for the antidepressant action of sleep deprivation. Thus, slow wave activity (SWA) in NREM sleep would be inversely related to the severity of depression. The model predicts that a number of sleep disturbances, including increased sleep latency, NREM sleep deficiency, shortening of REMOL, and disrupted sleep continuity, have broad consequences for cyclical regulation of bodily processes. Clinical evidence cited by Borbély and his coworkers has been used to support the main premise of the model, i.e., that disrupted sleep is closely linked to pathological diurnal changes in mood (49).

Borderland and colleagues recently revised the two-process model. The SCN is now viewed as orchestrating and integrating rhythms rather than simply generating them. Homeostasis of brain function may be achieved through periodic sleep/wake alternation, which itself is generated by either an underlying neuronal oscillator outside the SCN or by a behavioral relaxation oscillator as conceived in Process S. The SCN slowly advances or delays transiently and experimentally shifted human sleep/wake cycles towards the unshifted circadian melatonin rhythm in temporal isolation.

When orexinergic wake promotion is under circadian control and reflects the daily rise in Process C, this would be fully compatible with the two-process model. 
Sleep has a large effect on peripheral circadian oscillators due to such factors as the absence of locomotion, reduced body temperature, increased growth hormone and reduced glucocorticoid secretion (50).

The time spent in SWS is affected more by earlier waking than by the circadian phase, and is a major factor affecting sleep depth during the first third of the night. SWS reduction could thus increase the opportunity for REM sleep to express itself earlier in the night (50). In the context of the S-deficiency model, reduced REMOL is thus understood as being the result of a faulty homeostatic process rather than a phase advance of the endogenous circadian system. This hypothesis implies that sleep deprivation should exert its antidepressant effect by increasing the level of the homeostatic drive for sleep. The model thus predicts that sleep deprivation should result in increased levels of delta-wave activity during the recovery sleep period (38).

According to the S-deficiency model, an inappropriate increase in sleep pressure during wakefulness in patients with depression would result in complications with sleep initiation and sleep maintenance, and consequently in reduced SWS. The Sdeficiency model also provides a potential explanation for several depressionassociated phenomena, such as the leveling off of growth hormone secretion before bedtime, irregularities in the diurnal rhythms of plasma melatonin levels, and disturbed secretion of the hypothalamic-pituitary-adrenal (HPA) axis hormones (47).

Critiques of models of depression resulting from REM abnormalities (51-54) Although less elaborately developed than other models considered in this review, the rejection of an REM abnormality as key to depression questions some of the widely accepted assumptions about the causal importance of REM sleep 
abnormalities in individuals with depression. These assumptions relate to observations that individuals with depression exhibit abnormal REM sleep profiles somewhat resembling the patterns shown by healthy individuals during extended sleep, and additional observations that REM sleep deprivation has antidepressant effects (34). It has been argued that the relevance of these observations to depression is not firmly established. Furthermore, the clinical effects resulting from influencing the sleep/wake cycle or light therapy may occur as a result of their influence on specific irregularities that lie beneath sleep disturbances in mood disorders (33). A final argument for this viewpoint is that changes in the clinical status of patients with depression suggest that mood disorders might be triggered by chronobiological irregularities. Consequently, the relationship of these disturbances to the overall pathology is not yet established, and whether they are a cause or consequence of disturbances in other system has yet to be verified.

\section{The social rhythms hypothesis of depression}

The social rhythms hypothesis of depression was initially proposed by Ehlers, Monk, and colleagues in the late 1980 s $(55,56) \cdot(57,58)$. The hypothesis highlights the role of altered social rhythms in the etiology of depression and associated circadian rhythm dysregulation. The hypothesis proposes that susceptible individuals show more severe circadian and sleep disturbances with the disruption of social rhythms. Furthermore, the resulting disruption of nonphotic zeitgebers produces a chain of causation resulting in the entrainment of physiological circadian rhythms which in turn triggers depressive episodes. 
The hypothesis is supported by research that shows that social rhythms are disrupted and irregular in patients with mood disorders. Increased regularity of social rhythms is associated with improved sleep quality and less severity of depressive symptoms. However, there is limited evidence for an extension of this hypothesis, i.e., that disruption of social rhythms additionally disrupts other physiological cycles in patients with depression.

Social entrainment begins soon after birth such that an infant's eating activity and sleep schedules become synchronized with the home routines of the parents. Later entrainment influences include the schedules of watching television shows, sports and recreational activities, socializing with peers, and academic commitments. Once these social rhythms are established, cognitive structuring of an individual's day is established. This structuring can potentially become a zeitgeber in its own right and can maintain daily circadian rhythms.

Considerable evidence exists that social zeitgebers are powerful entraining agents of the human circadian system, and that a loss or change in social zeitgebers can disrupt the circadian system (56). In susceptible individuals, social rhythm irregularities may prompt mood episodes as a result of reducing exposure to social zeitgebers. Life events that directly alter daytime habits, e.g., retirement, grief, the birth of a child, jet lag, and shift work, are especially likely to generate a depressive episode (57).

Monk et al. developed an assessment procedure method called the social rhythm metric (SRM) (56). This instrument produces scores that are approximately Gaussian in their distribution and show good test-retest reliability. Specifically developed as a tool to explore the role of social zeitgebers in the etiology of depression, the authors 
anticipated that it would be useful for linking biological theories with previous social theories of depression. The investigators' work has shown that individuals may vary in the longitudinal course of their social rhythms in significant ways, and that some individuals may have stable SRM scores followed by a brief disruption and a return to the initial pattern. Others may have stable scores followed by a disruption, which then establishes a new social rhythm. Others may stay in the disrupted pattern over time so that it becomes the customary pattern of the individual.

Frank and colleagues (59) developed interpersonal and social rhythm therapy (IPSRT), an intervention program to repair and merge social rhythm regularity as a protective strategy against relapse into depressed states. Their justification was that mood disorders result from a complex interaction between genetic predisposition, psychosocial stressors, and circadian rhythmicity, which can have positive or negative effects on adherence to pharmacotherapy. The first step in IPSRT is to identify irregular social rhythms (e.g., sleep/wake schedules, meal times, exercise times, and irregular occupational schedules such as shift work and unemployment) using the SRM. Based on the information gained in this first step, maintenance goals are then identified and applied. Typical goals include the establishment of regular bed, wake, and meal times, switching to a more regular work schedule, and integrating a regular daily exercise session.

IPSRT has been most widely used in patients with bipolar, in whom it reduces the risk of symptom recurrence. Other evidence has suggested that it also effectively regulates social rhythms, accelerates remission in patients with depression, and lowers relapse rates compared with intensive pharmacotherapy. These observations 
merit a more direct investigation of IPSRT as an add-on or monotherapy in major depression.

\section{Chronotherapeutics}

Chronotherapeutic treatments, such as light therapy (LT), sleep deprivation, and sleep phase advance (SPA), are non-invasive treatments that take advantage of the body's health restorative capacities. These interventions are now being used to treat sleep and mood disorders by influencing the circadian rhythm of patients. In the majority of studies investigating these treatments, patients were treated with daily bright light (5000-10,000 lux) in the morning for 2-4 weeks, with each session lasting 30-60 min. The timing of the therapy depends on the patient's original chronotype, i.e., whether a morningness or eveningness subtype. Nonetheless, one meta-analysis has shown that the strategically timed application of light therapy has outcomes that are comparable to pharmacological treatment (60). Table 2 summarizes currently existing chronotherapeutic regimens for depression (61).

Table 2. Chronotherapeutic regimens to treat depression

\begin{tabular}{|l|c|}
\hline \multicolumn{1}{|c|}{ Duration of response to chronotherapy } & Type of applied therapy \\
\hline Days & total sleep deprivation TSD \\
\hline Weeks & partial sleep deprivation PSD \\
& repeated TSD OR PSD \\
\hline & repeated TSD OR PSD with antidepressant \\
\hline & phase advance of sleep cycle \\
\hline Months & TSD followed by sleep advance \\
\hline & single or repeated TSD OR PSD followed by light therapy \\
& $\begin{array}{c}\text { single or repeated TSD OR PSD followed by light therapy and } \\
\text { phase advance }\end{array}$ \\
\hline & lingle or repeated TSD OR PSD combined with lithuim or SSRI \\
& light therapy \\
\hline
\end{tabular}




\section{Conclusions}

Whether dysregulated circadian rhythms can cause depression or whether depression leads to variations in circadian rhythms is still unclear. Nevertheless, there is substantial clinical and observational evidence of a correlation existing between the two, and most people with depressed mood also have circadian rhythm irregularities.

Whether there is a causative link between circadian rhythm disruption and depression is also not yet fully confirmed. Nonetheless, the finding that some forms of depression respond to chronotherapeutic treatment, is consistent with the inference that circadian dysregulation seen in patients with depression may be a core component of depressive pathophysiology. There is now emerging evidence for a relationship between mood disorders and polymorphic variants of clock genes. Various circadian clock genes have now been proposed as key factors in the control of the endogenous time-keeping system, and abnormalities in the function of these elements may be involved in circadian alterations occurring in major depression.

Further evidence from continued investigations of these issues may promote a deeper appreciation of the contribution of circadian disturbances to the pathophysiology of mood disorders, and will hopefully yield improved therapeutic approaches for their treatment. 
Acknowledgements 


\section{References}

Inouye ST, Kawamura H. Persistence of circadian rhythmicity in a mammalian hypothalamic "island" containing the suprachiasmatic nucleus. Proceedings of the National Academy of Sciences of the United States of America. 1979 Nov;76(11):5962-6.

Reiter RJ. The melatonin rhythm: both a clock and a calendar. Experientia. 2. 1993;49(8):654-64.

Turek FW. From circadian rhythms to clock genes in depression. International Clinical Psychopharmacology. 2007;22:S1-S8.

van den Pol AN. The hypothalamic suprachiasmatic nucleus of rat: intrinsic

4. anatomy. Journal of Comparative Neurology. 1980;191(4):661-702.

Schibler U. Interaction Between Central and Peripheral Clocks in Mammals.

5. Biological Timekeeping: Clocks, Rhythms and Behaviour: Springer; 2017. p. 337-63. Hermanstyne TO, Simms CL, Carrasquillo Y, Herzog ED, Nerbonne JM. Distinct Firing Properties of Vasoactive Intestinal Peptide-Expressing Neurons in the Suprachiasmatic Nucleus. Journal of biological rhythms. 2016;31(1):57-67. Brown GM, McIntyre RS, Rosenblat J, Hardeland R. Depressive disorders: Processes leading to neurogeneration and potential novel treatments. Progress in Neuro-Psychopharmacology and Biological Psychiatry. 2017.

Mrosovsky N, Hattar S. Impaired masking responses to light in melanopsinknockout mice. Chronobiology international. 2003;20(6):989-99.

Berson DM, Dunn FA, Takao M. Phototransduction by retinal ganglion cells that set the circadian clock. Science. 2002;295(5557):1070-3.

Moore RY, Speh JC. Serotonin innervation of the primate suprachiasmatic 10 nucleus. Brain research. 2004;1010(1):169-73.

Stehle JH, von Gall C, Korf HW. Melatonin: a clock-output, a clock-input.

Journal of neuroendocrinology. 2003 Apr;15(4):383-9.

Richter CP. Biological Clocks in Medicine and Psychiatry: Shock-Phase 12.

Hypothesis. Proceedings of the National Academy of Sciences of the United States of America. 1960 Nov;46(11):1506-30.

Cuesta M, Cermakian N, Boivin DB. Circadian clock genes and psychiatric. The 13. Genetic Basis of Sleep and Sleep Disorders. 2013:351.

Bollettini I, Melloni EM, Aggio V, Poletti S, Lorenzi C, Pirovano A, et al. Clock genes associate with white matter integrity in depressed bipolar patients. Chronobiol Int. 2017;34(2):212-24.

Dallaspezia S, Locatelli C, Lorenzi C, Pirovano A, Colombo C, Benedetti F. 15.

Sleep homeostatic pressure and PER3 VNTR gene polymorphism influence antidepressant response to sleep deprivation in bipolar depression. Journal of affective disorders. 2016 Mar 01;192:64-9.

Darien I. International classification of sleep disorders. American Academy of Sleep Medicine. 2014.

Hasler G, Drevets WC, Manji HK, Charney DS. Discovering endophenotypes 16.

for major depression. Neuropsychopharmacology : official publication of the American College of Neuropsychopharmacology. 2004 Oct;29(10):1765-81. 
Matsubara T, Matsuo K, Harada K, Nakano M, Nakashima M, Watanuki T, et 18. al. Distinct and Shared Endophenotypes of Neural Substrates in Bipolar and Major Depressive Disorders. PloS one. 2016;11(12):e0168493.

Misiak B, Frydecka D, Rybakowski JK. Editorial: Endophenotypes for 19. Schizophrenia and Mood Disorders: Implications from Genetic, Biochemical, Cognitive, Behavioral, and Neuroimaging Studies. Frontiers in psychiatry. 2016;7:83. Dudley TE, DiNardo LA, Glass JD. Endogenous regulation of serotonin release 20. in the hamster suprachiasmatic nucleus. Journal of Neuroscience. 1998;18(13):504552.

Pontes ALBd, Engelberth RCGJ, Nascimento Jr EdS, Cavalcante JC, Costa 21.

MSMdO, Pinato L, et al. Serotonin and circadian rhythms. Psychology \& Neuroscience. 2010;3(2):217-28.

Takeuchi K, Mohammad S, Ozaki T, Morioka E, Kawaguchi K, Kim J, et al. 22. Serotonin-2C receptor involved serotonin-induced $\mathrm{Ca} 2+$ mobilisations in neuronal progenitors and neurons in rat suprachiasmatic nucleus. Scientific reports. 2014;4. Mansour HA, Wood J, Logue T, Chowdari KV, Dayal M, Kupfer DJ, et al. 23. Association study of eight circadian genes with bipolar I disorder, schizoaffective disorder and schizophrenia. Genes, brain, and behavior. 2006 Mar;5(2):150-7. Albrecht U. Circadian clocks and mood-related behaviors. Handbook of 24. experimental pharmacology. 2013(217):227-39.

Chung S, Lee EJ, Yun S, Choe HK, Park S-B, Son HJ, et al. Impact of circadian 25. nuclear receptor REV-ERB $\alpha$ on midbrain dopamine production and mood regulation. Cell. 2014;157(4):858-68.

Shi J, Wittke-Thompson JK, Badner JA, Hattori E, Potash JB, Willour VL, et al. 26. Clock genes may influence bipolar disorder susceptibility and dysfunctional circadian rhythm. American Journal of Medical Genetics Part B: Neuropsychiatric Genetics. 2008;147(7):1047-55.

Van Cauter E, Turek FW. Depression: a disorder of timekeeping? Perspectives in biology and medicine. 1986;29(4):510-20.

Association AP. Diagnostic and statistical manual of mental disorders (DSM- 28. $\left.5^{\circledR}\right)$ : American Psychiatric Pub; 2013.

Ohayon MM. Insomnia: a ticking clock for depression? : Pergamon; 2007. 29. David Nutt D, Paterson L. Sleep disorders as core symptoms of depression. 30. Dialogues Clin Neurosci. 2008;10(3):329-36.

Armitage R. Microarchitectural findings in sleep EEG in depression: diagnostic implications. Biological Psychiatry. 1995;37(2):72-84.

Pandi-Perumal SR, Moscovitch A, Srinivasan V, Spence DW, Cardinali DP, 32. Brown GM. Bidirectional communication between sleep and circadian rhythms and its implications for depression: lessons from agomelatine. Progress in neurobiology. 2009;88(4):264-71.

Armitage R, Hoffmann R, Trivedi M, Rush AJ. Slow-wave activity in NREM 33. sleep: sex and age effects in depressed outpatients and healthy controls. Psychiatry research. 2000;95(3):201-13.

Van den Hoofdakker R. Chronobiological theories of nonseasonal affective 34. disorders and their implications for treatment. Journal of Biological Rhythms. 1994;9(2):157-83. 
Ben-Hamo M, Larson TA, Duge LS, Sikkema C, Wilkinson CW, Horacio O, et al.

Circadian Forced Desynchrony of the Master Clock Leads to Phenotypic

Manifestation of Depression in Rats. eneuro. 2016;3(6):ENEURO. 0237-16.2016.

Borbély AA. A two process model of sleep regulation. Human neurobiology. 36

1982.

Kripke DF, Elliott JA, Welsh DK, Youngstedt SD. Photoperiodic and circadian 37.

bifurcation theories of depression and mania. F1000Research. 2015;4.

Daan S, Beersma D, Borbély AA. Timing of human sleep: recovery process 38 .

gated by a circadian pacemaker. American Journal of Physiology-Regulatory, Integrative and Comparative Physiology. 1984;246(2):R161-R83.

Monteleone P, Maj M. The circadian basis of mood disorders: recent 39. developments and treatment implications. European Neuropsychopharmacology. 2008;18(10):701-11.

Cervantes P, Gelber S, Ng YK. Circadian secretion of cortisol in bipolar 40. disorder. Journal of psychiatry \& neuroscience: JPN. 2001;26(5):411.

Nurnberger JI, Adkins S, Lahiri DK, Mayeda A, Hu K, Lewy A, et al. Melatonin 41. suppression by light in euthymic bipolar and unipolar patients. Archives of general psychiatry. 2000;57(6):572-9.

Landgraf D, McCarthy MJ, Welsh DK. The role of the circadian clock in animal 42. models of mood disorders. Behavioral neuroscience. 2014;128(3):344.

Wehr TA, Wirz-Justice A. Internal coincidence model for sleep deprivation 43. and depression. Sleep. 1980;26:33.

St Hilaire MA, Gooley JJ, Khalsa SBS, Kronauer RE, Czeisler CA, Lockley SW. 44. Human phase response curve to a $1 \mathrm{~h}$ pulse of bright white light. The Journal of physiology. 2012;590(13):3035-45.

Germain A, Kupfer DJ. Circadian rhythm disturbances in depression. Human Psychopharmacology: Clinical and Experimental. 2008;23(7):571-85.

Reinberg A, Ashkenazi I. Internal desynchronization of circadian rhythms and tolerance to shift work. Chronobiology international. 2008;25(4):625-43.

Boivin DB. Influence of sleep-wake and circadian rhythm disturbances in 47. psychiatric disorders. Journal of Psychiatry \& Neuroscience. 2000.

Chellappa SL, Schröder C, Cajochen C. Chronobiology, excessive daytime 48. sleepiness and depression: Is there a link? Sleep medicine. 2009;10(5):505-14. Borbély A. The S-deficiency hypothesis of depression and the two-process 49. model of sleep regulation. Pharmacopsychiatry. 1987;20(01):23-9.

Borbély AA, Daan S, Wirz-Justice A, Deboer T. The two-process model of sleep regulation: a reappraisal. Journal of sleep research. 2016.

BERGER M, RIEMANN D. REM sleep in depression-an overview. Journal of

45. Sleep Research. 1993;2(4):211-23.

Monteleone P, Maj M. Circadian rhythm disturbances in depression: 52. implications for treatment and quality of remission. Medicographia. 2009;31(2):1329.

Adrien J. Neurobiological bases for the relation between sleep and 53 . depression. Sleep medicine reviews. 2002;6(5):341-51.

Akiskal HS. Mood disturbances. The medical basis of psychiatry: Springer; 2016. p. 459-75.

54. 
Ehlers CL, Frank E, Kupfer DJ. Social zeitgebers and biological rhythms: a 55 . unified approach to understanding the etiology of depression. Archives of general psychiatry. 1988;45(10):948-52.

Monk TK, Flaherty JF, Frank E, Hoskinson K, Kupfer DJ. The Social Rhythm 56. Metric: An instrument to quantify the daily rhythms of life. Journal of Nervous and Mental Disease. 1990.

Monk TH, Kupfer DJ, Frank E, Ritenour AM. The Social Rhythm Metric (SRM): 57. measuring daily social rhythms over 12 weeks. Psychiatry research. 1991;36(2):195207.

Monk TH. Enhancing circadian zeitgebers. Sleep. 2010;33(4):421. 58.

Frank E, Swartz HA, Kupfer DJ. Interpersonal and social rhythm therapy: 59. managing the chaos of bipolar disorder. Biological psychiatry. 2000;48(6):593-604. Golden RN, Gaynes BN, Ekstrom RD, Hamer RM, Jacobsen FM, Suppes T, et al. 60 The efficacy of light therapy in the treatment of mood disorders: a review and metaanalysis of the evidence. American Journal of Psychiatry. 2005;162(4):656-62.

Wirz-Justice A, Benedetti F, Berger M, Lam RW, Martiny K, Terman M, et al. 61. Chronotherapeutics (light and wake therapy) in affective disorders. Psychological medicine. 2005;35(07):939-44. 